Objectives The aim is to examine occupational noise exposure as a risk factor for depression, utilising noise exposure as an objective measure of distressing working conditions that circumvents reporting bias.

Method In a 7-year cohort study we followed 109378 industrial workers and 45613 financial workers from 2001 or first year of employment thereafter until 2007. At start and end of follow up we recorded mean, full-shift noise exposure levels by personal dosimeters for 1077 workers from randomly selected companies. We assumed a linear relation with calendar year and predicted exposure levels by trade and occupation since 1980 and calculated cumulative noise exposure. Danish national registries provided complete employment histories since 1980, psychiatric diagnoses (1977-2001), and redemption of antidepressants (Selective Serotonin Reuptake Inhibitors, SSRI) (1994-2007). Workers with psychiatric diagnoses or use of antidepressants before 2001 were excluded.

Results During follow-up we identified 7754 incident users of SSRIs. Among women, risk of starting SSRI medication increased by cumulative noise exposure level OR=1.02 (95\% CI: $1.01-$ 1.02) per $\mathrm{dB}(\mathrm{A})$-year when adjusted for age, calendar year and socioeconomic status. When excluding white-collar workers no effect was seen among women and no effect of noise was apparent among men overall.

Conclusions These preliminary results do not provide strong evidence that occupational noise exposure is a risk factor for depression. The increased OR seen among all women can be explained by differences in socioeconomic status between the blue- collar industrial workers and the white-collar financial workers since no trends were apparent in internal analyses among blue-collar workers.

\section{ASSOCIATIONS BETWEEN PRE-DEFINED OCCUPATIONAL JOB TASKS AND BREAST CANCER RISK}

${ }^{1}$ Sylvia Rabstein, ${ }^{1}$ Beate Pesch, ${ }^{2}$ Volker Harth, ${ }^{3}$ Christina Justenhoven, ${ }^{4}$ Ute Hamann, ${ }^{3}$ Hiltrud Brauch, ${ }^{5}$ Yon Ko, ${ }^{1}$ Thomas Bruening. ${ }^{1}$ Institute of Prevention and Occupational Medicine, German Social Accident Insurance (IPA), Bochum, Germany; ${ }^{2}$ Institute for Occupational Medicine and Maritime Medicine, University Medical Centre Hamburg-Eppendorf, Hamburg, Germany; ${ }^{3}$ Dr. Margarete Fischer-Bosch-Institute of Clinical Pharmacology and University of Tübingen, Stuttgart, Germany; ${ }^{4}$ Molecular Genetics of Breast Cancer, Deutsches Krebsforschungszentrum (DKFZ), Heidelberg, Germany; ${ }^{5}$ Department of Internal Medicine, Evangelische Kliniken Bonn gGmbH, Johanniter-Krankenhaus Bonn, Bonn, Germany

\subsection{6/oemed-2014-102362.261}

Objectives The role of occupational exposures in agricultural and industrial settings has been addressed in several breast cancer studies. Recently, the influence of shift work in nurses added as an occupational hazard that has been intensively discussed. Here, we investigate the association of job tasks in the industrial and health sector and breast cancer in a large case-control study. Method The population-based case-control study Gene-ENvironment Interaction and Breast CAncer (GENICA) was conducted in the Greater Region of Bonn, Germany. Occupational history and job task information were collected in computer-assisted interviews. Thirty pre-defined job tasks were assessed for 1143 cases and 1155 controls in addition to the occupational history. Risk estimates were calculated as odds ratios (ORs) with 95\% confidence intervals (CIs) conditional on age and adjusted for potential confounders.

Results First preliminary results indicate an increased ageadjusted risk for women who ever worked in anaesthesia (OR
1.87; 95\% CI 1.03-8.0), based on fourteen cases and five controls.

Conclusions Our study revealed an increased risk for ever working in anaesthesia. This elevated risk might origin from chemical exposures or night shift work. Interactions between exposures and night work might be relevant in the progression of breast cancer. However, the results of this study are limited by the low prevalence of risk jobs and specific exposures.

\section{GENDER BIAS IN OCCUPATIONAL EPIDEMIOLOGY RESEARCH: A SYSTEMATIC REVIEW ON WORK-RELATED LUNG CANCER}

${ }^{1}$ Charles-Olivier Betansedi, 1,2Emilie Counil. 'Giscop 93, Université Paris 13, Bobigny, Ile-de-France, France; ${ }^{2}$ EHESP Rennes, Sorbonne Paris Cité, Paris, Ile-de-France, France; ${ }^{3}$ IRIS (UMR 8156-997), Université Paris 13, Bobigny, Ile-de-France, France

\subsection{6/oemed-2014-102362.262}

Objectives The "one-eyed science" pointed out by some authors has contributed to the invisibilization of working conditions as a health determinant among women. Our objectives were to document current epidemiological practices in the assessment of work-related lung cancer risks, and to discuss how genderrelated biases compromise the scientific validity of exposure and risk estimates among women, as compared to men.

Method A systematic literature review over the last 7 years was performed, and based on the screening of 410 abstracts retrieved from PubMed, 122 articles were retained. Data were collected through a questionnaire, and analysed both quantitatively and qualitatively. Articles were classified according to the gender distribution of the study sample as either men only, women only or mixed.

Results Androcentrism was present, as nearly 50\% of studies recruited men-only participants. Moreover, $45 \%$ of them were subject to an overgeneralization of study results. Gender-insensitivity could be observed from the papers (35\%) which did not provide justification for the gender composition of study sample. A double standard was also suspected in the exposure assessment methods. Sex and gender-related terms were found to be frequently used interchangeably.

Conclusions Upgraded results with an increased sample size are forthcoming. Meanwhile, these preliminary results raise the question of the "gender bias" in epidemiology, and how sex and gender should be taken into account in the design, conduct, analysis and dissemination of results in order to minimise genderrelated biases and reinforce the scientific validity of research.

\section{APPLICATION OF A DYNAMIC POPULATION-BASED MODEL TO ASSESS THE EFFECT OF SILICA EXPOSURE INTERVENTIONS ON COPD IN DUTCH CONSTRUCTION WORKERS: RESULTS FROM THE 'RELIEVED WORKING STUDY'}

${ }^{1}$ Anjoeka Pronk, ${ }^{1}$ Ruud Boessen, ${ }^{1,2}$ Erik van Deurssen, ${ }^{1}$ Tim Meijster, ${ }^{1}$ Rinke Klein Entink, ${ }^{1}$ Birgit van Duuren-Stuurman, ${ }^{2}$ Dick Heederik, ${ }^{3}$ Nick Warren, ${ }^{3}$ Emma Tan. ${ }^{1} T N O$, Zeist, The Netherlands; ${ }^{2}$ IRAS, Utrecht, The Netherlands; ${ }^{3} \mathrm{HSL}$, Buxton, UK

\subsection{6/oemed-2014-102362.263}

Objectives A multidimensional intervention aimed at reducing silica exposure in the Dutch construction industry was performed. The objective of this study was to assess the effect of 
the achieved reduction in exposure on the burden of chronic obstructive pulmonary disease (COPD) in construction workers. Method The intervention aimed at technical, organisational and psycho-social factors and was performed in four construction companies. Pre and post intervention respirable quartz exposure measurements were taken in these and four additional control companies. A mathematical simulation model was used to generate COPD prevalences (GOLD stage $\geq 1$ ) in response to silica exposure, population characteristics and Dutch trends in smoking behaviour for a population of 20-65 year old construction workers with lifetime silica exposure.

Results Pre-intervention exposure assessment demonstrated highest respirable quartz levels $\left(\mathrm{mg} / \mathrm{m}^{3}\right)$ for concrete drillers (GM: 0.20, GSD: 2.75), tuck pointers (GM: 0.18, GSD (2.18) and demolishers (GM: 0.12, GSD: 2.86), exceeding the Dutch occupational exposure limit (OEL) in 71, 92 and 97\% of cases, respectively. Preliminary simulations estimated COPD prevalence at 21,20 and $17 \%$ for these groups respectively, as compared to $14 \%$ when quartz exposure is reduced to the Dutch OEL and $8 \%$ with no exposure.

Conclusions For several job categories high exposure levels exceeding the Dutch OEL were observed. Reducing these levels to below the OEL would lead to a substantial reduction in the burden of disease. The post intervention exposure levels will become available early 2014. The effect on the burden of disease and economic impact will be assessed with an refined model incorporating population dynamics.

\section{CARPAL TUNNEL SYNDROME AND CARPAL TUNNEL SYNDROME-LIKE SYMPTOMS IN RELATION TO MECHANICAL EXPOSURES ASSESSED BY A JOB EXPOSURE MATRIX: A TRIPLE CASE-REFERENT STUDY}

${ }^{1}$ Sorosh Tabatabaeifar, ${ }^{2}$ Susanne Wulff Svendsen, ${ }^{3}$ Birger Johnsen, ${ }^{3}$ Anders Fuglsang-Frederiksen, ${ }^{1}$ Poul Frost. 'Danish Ramazzini Centre, Department of Occupational Medicine, Aarhus University Hospital, Aarhus, Denmark; ${ }^{2}$ Danish Ramazzini Centre, University Department of Occupational Medicine, Herning Regional Hospital, Herning, Denmark; ${ }^{3}$ Department of Neurophysiology, Aarhus University Hospital, Aarhus, Denmark

\subsection{6/oemed-2014-102362.264}

Objectives To evaluate relations between occupational mechanical exposures and (1) carpal tunnel syndrome verified by ENG (ENG+CTS) and (2) CTS-like symptoms with normal ENG (ENG-CTS).

Method We plan a triple-case referent study of 1000 ENG + CTS and 1000 ENG-CTS cases identified at a university department of clinical neurophysiology. For each case, two sex, age, and primary care centre matched controls will be sampled (risk set sampling). Both retrospectively and prospectively identified cases will be included with standardised clinical examination of the last-mentioned group. Conditional logistic regression analyses will be performed comparing the two case control sets, while unconditional logistic regression will be applied comparing ENG+CTS cases to ENG-CTS controls. Questionnaire information will be collected on job history, lifestyle, symptoms, and disability. Job titles will be linked to a job exposure matrix (JEM) based on measurements of hand-wrist movements (goniometer measurements) and expert ratings.

Results The main hypothesis is that exposure-response relations will be found for ENG+CTS, but not for ENG-CTS with respect to forceful work and awkward wrist postures, while repetitive work will show exposure-response relations in both groups. The Danish Working Environment Research Fund has granted financial support for a 3 -year $\mathrm{PhD}$ project starting January 2014.

Conclusions The study will take advantage of specific and well documented case diagnoses and independent exposure assessment. The results are expected to produce new insights into exposure-response relations between occupational mechanical exposures and risk of CTS.

\section{ARE INDOOR CONCENTRATIONS OF AIRBORNE MOULD SPORES IN NON-INDUSTRIAL ENVIRONMENTS SUFFICIENTLY HIGH TO CAUSE HYPERSENSITIVITY PNEUMONITIS?}

${ }^{1}$ Henrik A Kolstad, ${ }^{2}$ Simon Skov, ${ }^{3}$ Tine Birgitte Halsen Malling, ${ }^{4}$ Elisabeth Bendstrup, 1,5 Vivi Schlünssen. ${ }^{1}$ Department of Occupational Medicine, Danish Ramazzini Centre, Aarhus University Hospital, Aarhus, Denmark; ${ }^{2}$ Forest and Landscape, Faculty of Life Sciences, University of Copenhagen, Copenhagen, Denmark; ${ }^{3}$ Department of Occupational Medicine, Danish Ramazzini Centre, Aalborg University Hospital, Aalborg, Denmark; ${ }^{4}$ Department of Respiratory Medicine and Allergology, Aarhus University Hospital, Aarhus, Denmark; ${ }^{5}$ Department of Public Health, Section for Environment, Work, and Health, Danish Ramazzini Centre, Aarhus University, Aarhus, Denmark

\subsection{6/oemed-2014-102362.265}

Objectives Antigen exposure is the only diagnostic criteria specific for hypersensitivity pneumonitis (HP) compared with other interstitial lung diseases. Indoor mould exposure in non-industrial environments has been claimed to cause HP, but little is known about exposure levels. Our objective was to compare indoor concentrations of airborne mould spores for patients diagnosed with indoor HP with background levels and levels measured for patients diagnosed with farmers' lung and suberosis.

Method We included 8 patients diagnosed with HP based on characteristic clinical findings, signs of indoor mould growth at home or at their non-industrial work place, and increased levels of precipitating antibodies for moulds. We collected 110 air samples from all affected rooms, adjacent rooms, and outdoor.

Results The average total spore concentrations varied between 22000 and 36000 spores per $\mathrm{m}^{3}$, and the average viable concentrations between $35 \mathrm{CFU} / \mathrm{m}^{3}$ and $457 \mathrm{CFU} / \mathrm{m}^{3}$, with no clear association between spore concentration and affected rooms, adjacent rooms and outdoor.

Conclusions The observed levels did not exceed usual indoor and outdoor levels and were orders of magnitude below levels measured for patients diagnosed with farmers' lung and suberosis, and we question if indoor mould levels in non-industrial environments are sufficient to cause HP. Relying solely on signs of moulds or presence of precipitating antibodies when diagnosing HP may cause other interstitial lung diseases to be overseen and patients may take initiatives such as quitting the job or leaving their homes that will not alleviate their disease but have significant social consequences.

\section{PROFESSIONAL TRAJECTORY IS ASSOCIATED WITH MULTIPLE CARCINOGENIC EXPOSURES AT WORK AMONG MEN : DATA FROM A COHORT OF PATIENTS SUFFERING RESPIRATORY CANCER}

${ }^{1,2}$ Emilie Counil, ${ }^{3,4}$ Mélanie Bertin, ${ }^{3}$ The Giscop93 team. ${ }^{1}$ EHESP Rennes, Sorbonne Paris Cité, Paris, France; ${ }^{2}$ IRIS, UMR 8156-997, Bobigny, France; ${ }^{3}$ IRSET, UMR Inserm 1085, Rennes, France; ${ }^{4}$ Giscop93, Université Paris 13, Bobigny, France

10.1136/oemed-2014-102362.266 